\title{
Preparation and characterization of nanocomposite films from oil palm pulp nanocellulose / poly (Vinyl alcohol) by casting method
}

\begin{abstract}
TEMPO-oxidize nanocellulose (TONC) suspension has been obtained from total chlorine free (TCF) oil palm empty-fruit-bunches (OPEFB) pulp using 4-acetamido-TEMPO (2,2,6,6tetramethyl piperidin-1-oxyl) mediated oxidation with sodium hypochlorite and sodium bromide in water at $25^{\circ} \mathrm{C}$ and $\mathrm{pH} 10$. TONC suspension with varied content from 0.5 to $6 \%$ $(\mathrm{w} / \mathrm{w})$ reinforced polyvinyl alcohol (PVA) polymer based nanocomposite films were prepared by the casting method. The structural interaction between the TONC and PVA was characterized by the Fourier transform infrared (FT-IR) spectroscopy, nuclear magnetic resonance (NMR) spectroscopy, X-ray diffraction (XRD) and scanning electron microscopy (SEM). It was found that the 4\% (w/w) TONC content reinforced nanocomposite exhibited the highest tensile strength and modulus with an increase of $122 \%$ and $291 \%$ respectively, compared to PVA while the elongation at break decreased about $42.7 \%$. Thermal stability of PVA based nanocomposite films was improved after incorporation of TONC. Incorporation of TONC in PVA film increases its crystallinity due to strongly linking between the hydroxyl groups of materials however considerable decreases beyond $2 \mathrm{wt} \%$ loading are observed. TONC incorporation beyond $2 \mathrm{wt} \%$ also reduces the melting temperature peaks and enthalpy of nanocomposite films. FT-IR spectra, NMR and SEM indicate that there is interaction between the TONC and PVA.
\end{abstract}

Keyword: SOil palm pulp nanocellulose; Nanocomposite films; Poly (vinyl) alcohol; TEMPO-oxidation; Thermal analysis 